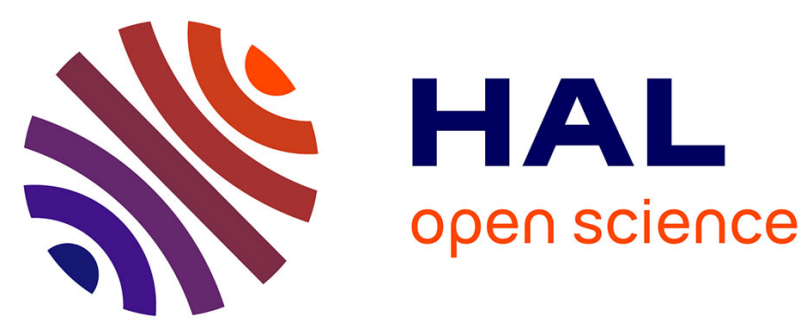

\title{
Analyse de la répartition du volume des pores dans les assemblages argile-squelettes : comparaison entre un modèle d'espace poral textural et les données fournies par la porosimétrie au mercure \\ Jean-Claude Fies, Claude Jeandet
}

\section{To cite this version:}

Jean-Claude Fies, Claude Jeandet. Analyse de la répartition du volume des pores dans les assemblages argile-squelettes : comparaison entre un modèle d'espace poral textural et les données fournies par la porosimétrie au mercure. Agronomie, 1984, 4 (9), pp.891-899. hal-00884710

\author{
HAL Id: hal-00884710 \\ https://hal.science/hal-00884710
}

Submitted on 1 Jan 1984

HAL is a multi-disciplinary open access archive for the deposit and dissemination of scientific research documents, whether they are published or not. The documents may come from teaching and research institutions in France or abroad, or from public or private research centers.
L'archive ouverte pluridisciplinaire HAL, est destinée au dépôt et à la diffusion de documents scientifiques de niveau recherche, publiés ou non, émanant des établissements d'enseignement et de recherche français ou étrangers, des laboratoires publics ou privés. 


\section{Analyse de la répartition du volume des pores dans les assemblages argile-squelettes : compa- raison entre un modèle d'espace poral textural et les données fournies par la porosimétrie au} mercure

Jean-Claude FIES

avec la collaboration technique de Claude JEANDET

I.N.R.A., Station de Science du Sol, Centre de Recherches d'Avignon, B.P. 91, F 84140 Montfavet frontant un modèle simple d'organisation texturale aux données fournies par la porosimétrie au mercure. Ces échantillons sont constitués du mélange, à des teneurs variées, d'une argile gonflante et d'un squelette constitué soit d'un limon $(2-20 \mu \mathrm{m})$, soit d'un sable $(100-200 \mu \mathrm{m})$. En admettant l'hypothèse d'une valeur constante de la masse volumique de la phase argileuse dans les mélanges, l'espace poral textural peut être divisé en deux compartiments :

- celui des pores lacunaires résultant du retrait de la phase argile entre les grains du squelette, à la dessiccation,

- celui des pores propres à la phase argile elle-même.

La forme des courbes cumulatives d'entrée de mercure confirme l'existence de ces deux compartiments de porosité. La première partie de la courbe correspond à l'occupation des pores lacunaires, la seconde à l'occupation partielle des pores de l'argile.

Pour tous les mélanges, une corrélation étroite est obtenue entre volumes de lacune calculé et mesuré. La valeur du diamètre correspondant au début de l'intrusion du mercure dans les pores de l'argile a été établie à $0,05 \mu \mathrm{m}$, sur la base des données obtenues avec les mélanges à squelette sableux.

Dans le cas des mélanges à squelette limoneux, l'hypothèse d'une confusion partielle entre pores lacunaires et pores propres à l'argile permet d'expliquer un écart systématique entre volumes de lacune calculé et mesuré sans remettre en cause le modèle général d'organisation texturale.

Mots clés additionnels :Porosimétrie à mercure, volume des pores, modèle d'organisation texturale. modelling.

The pore space fabric of soil was studied on dried artificial samples comparing a simple textural pore space model with an analysis based on mercury intrusion.

A swelling clay was mixed in various proportions with either a sandy skeleton $(100-200 \mu \mathrm{m})$ or a silty skeleton $(2-20 \mu \mathrm{m})$. Assuming a constant clay bulk density value for all mixtures, the pore space volume could be divided into :

- the lacunar pore space volume due to clay shrinkage between skeleton particles,

- the clay fabric pore space.

This division was apparently confirmed by the shape of the cumulative mercury intrusion curves. The first part of the curve could be interpreted as a complete mercury intrusion of the lacunar pore space and the second part as a partial mercury intrusion of the clay fabric.

For both silty-clay and sandy-clay mixtures, calculated and measured lacunar pore space were strongly correlated. The starting diameter of mercury intrusion in clay fabric was fixed at a value of $0.05 \mu \mathrm{m}$, from the data obtained on sandy-clay mixtures. For silty-clay mixtures, a systematic discrepancy between calculated and measured lacunar pore space could be explained by a slight variation of the clay bulk density, which did not call into question the general scheme of the model.

Additional key words : Mercury porosimetry, pore volume, soil fabric model. 


\section{INTRODUCTION}

Dans des travaux antérieurs, la porosité d'assemblage des particules du sol, ou porosité texturale, a été étudiée expérimentalement sur des échantillons obtenus par dessiccation du mélange d'une pâte argileuse et d'un squelette (FIES, 1971, 1978).

Rappelons que l'espace poral textural, à l'état sec, des mélanges argile-squelette a été alors divisé en :

- un espace poral propre à la phase argileuse,

- un espace poral lacunaire, lié au retrait de la phase argileuse entre les particules du squelette.

Cette analyse a été suggérée par les résultats des bilans de volurnes calculés sur la base des mesures de masse volumique des mélanges, $\rho_{\mathrm{d}}$, de masse volumique de la phase argileuse, $\rho_{\mathrm{a}}$.

Dans ces bilans, la valeur de $\rho_{\mathrm{a}}$, mesurée sur des échantillons secs de l'argile utilisée, a été supposée constante, c'est-à-dire indépendante de l'association argile-squelette, à titre d'hypothèse de travail.

La comparaison du volume encombré par $1 \mathrm{~g}$ de mélange, soit $1 / \rho_{\mathrm{d}}$, à la somme des volumes correspondant respectivement au volume de squelette et au volume encombré par la phase argileuse montre alors que cette somrne est toujours inférieure à $1 / \rho_{\mathrm{d}}$. Ceci peut s'écrire sous la forme suivante :

$$
1 / \rho_{d}>S / \rho_{s}^{s}+A / \rho_{a}
$$

dans laquelle $S$ et $A$ représentent les teneurs pondérales en sable et argile et $\rho_{s}^{s}$ la masse volumique de solide du squelette considéré.

Pour rétablir l'égalité entre les 2 termes de la relation ci-dessus, on était alors fondé à admettre la présence d'un volume $V_{1}$ de lacunes ou à infirmer l'hypothèse de la constance de la masse volumique de l'argile. Il faut noter dans la $2^{\mathrm{e}}$ hypothèse que la nonexistence d'une phase lacunaire implique un accroissement très important de la porosité de la phase argile.

L'observation de lames minces, réalisées dans les seuls mélanges sable-argile, a confirmé la présence d'un volume lacunaire. Par ailleurs, CHRETIEN (1979) a présenté de nombreuses illustrations de pores lacunaires observés en lames minces dans des mélanges de sables, à morphologies variées, et d'argile. Cet auteur note cependant un écart entre volumes de pores lacunaires calculés et mesurés sans pouvoir conclure, du fait de l'imprécision de la méthode d'analyse des photographies, à une variation conséquente de la valeur de $\rho_{a}$.

Enfin, la présence d'une phase lacunaire dans les mélanges après séchage apparaît cohérente avec l'existence du point d'entrée d'air, sur les courbes de retrait dites de $1^{\text {re }}$ organisation (STENGEL, 1981). Ce dernier a montré que le point d'entrée d'air se manifestait à des valeurs du potentiel de l'eau auxquelles la phase argileuse était toujours saturée en eau.

Le but du présent travail est de vérifier l'hypothèse de la constance de la masse volumique de la phase argileuse dans les mélanges et l'existence de 2 compartiments de volume poreux en utilisant une méthode directe de mesure du volume des pores : la porosimétrie au mercure.

Pour cela, 2 types de mélanges, caractérisés l'un par un squelette sableux, l'autre par un squelette limoneux, associés à des quantités variées d'une même fraction argileuse, ont été soumis, d'une part, à des mesures de masse volumique, d'autre part, à des mesures d'entrée de mercure.

\section{CONDITIONS EXPÉRIMENTALES}

\section{A. Echantillons étudiés}

\section{Caractéristiques et mode de préparation des échantillons}

Il s'agit d'échantillons réalisés par mélange à l'état humide d'une pâte argileuse et d'un squelette de sol, en proportions pondérales variées.

Chaque mélange est ensuite ressuyé sur buchner, par le vide produit par une trompe à eau, pendant $5 \mathrm{~h}$, puis desséché à l'atmosphère du laboratoire et enfin à l'étuve à $105^{\circ} \mathrm{C}$ (FIES \& STENGEL, 1981a). On obtient ainsi un massif poreux dépourvu de fissures d'environ $100 \mathrm{~g}$.

La pâte argileuse, constituée par la fraction inférieure à $2 \mu \mathrm{m}$ extraite de l'horizon d'accumulation d'un sol limoneux et rendue calcique, contient essentiellement des particules phylliteuses (tabl. 1).

Deux classes de squelette ont été utilisées. L'une correspond à un limon, séparé du même horizon par sédimentation entre 2 et $20 \mu \mathrm{m}$, l'autre à un sable de Fontainebleau, séparé par tamisage entre 100 et $200 \mu \mathrm{m}$.

\section{TABLEAU 1}

Caractéristiques des constituants des mélanges Characteristics of the particles used in mixture.

\begin{tabular}{|c|c|c|}
\hline Constituant & $\begin{array}{l}\text { Caractéristiques } \\
\left.\text { minéralogiques ( }{ }^{1}\right)\end{array}$ & $\begin{array}{l}\text { Masse volumique } \\
\text { de solide, } \mathrm{g} / \mathrm{cm}^{3}\end{array}$ \\
\hline Argile $<2 \mu \mathrm{m}$ & $\begin{array}{ll}\text { Smectite } & 40 \% \\
\text { Vermiculite } & \\
\text { Illite } & 25 \% \\
\text { Kaolinite } & 25 \% \\
\text { Oxydes de fer } & 10 \% \\
\text { Traces de quartz }\end{array}$ & 2,809 \\
\hline $\begin{array}{l}\text { Limon } \\
2-20 \mu \mathrm{m}\end{array}$ & $\begin{array}{l}\text { Quartz, } \\
\text { traces de feldspaths } \\
\text { et de muscovite }\end{array}$ & 2,700 \\
\hline $\begin{array}{c}\text { Sable } \\
100-200 \mu \mathrm{m}\end{array}$ & Quartz & 2,655 \\
\hline
\end{tabular}

(1) D'après TESSIER, 1975.

\section{Mesures des masses volumiques après séchage}

Le volume de chaque échantillon est déterminé après saturation dans le pétrole jusqu'à taux constant, ressuyage de l'excès de pétrole et mesure de la poussée d'Archimède dans le même liquide (MONNIER et al., 1973).

Compte tenu de la granulométrie des échantillons, les mesures de volume ont été conduites, pour les mélanges limoneux, sur des agrégats obtenus par tamisage entre 2 et $3,15 \mathrm{~mm}$ après fracturation de chacun des massifs poreux. 
Pour les mélanges sableux, le volume a été mesuré avec la même technique sur des fragments de 10 à $8 \mathrm{~cm}^{3}$ pour les échantillons contenant moins de 50 p. 100 d'argile, sur des fragments de 5 à $3 \mathrm{~cm}^{3}$ audelà de 50 p. 100 d'argile (FIES \& STENGEL, 1981a).

\section{Expression des résultats}

Les résultats sont exprimés en masse volumique, $\rho_{d}$, calculée après mesure du poids sec de l'échantillon soumis à la mesure de volume. Une étude de répétabilité a montré que les masses volumiques sont estimées à moins de 0,5 p. 100 près. En terme de porosité, cela signifie que la valeur moyenne de la porosité d'un mélange est estimée dans un intervalle inférieur à \pm 1 p. 100. Les masses volumiques des mélanges sont présentées au tableau 2.

Les volumes de pores sont exprimés en $\mathrm{cm}^{3}$ pour $100 \mathrm{~g}$ de mélange.

TABLEAU 2

Masse volumique des mélanges après séchage.

Bulk density of the dried clay-skeleton mixtures.

\begin{tabular}{|c|c|c|c|c|c|c|c|c|c|c|c|c|}
\hline Teneur pondérale en argile & 10 & 15 & 20 & 25 & 30 & 40 & 50 & 60 & 70 & 80 & 90 & 100 \\
\hline Squelette $2-20 \mu \mathrm{m}$ & 1,600 & 1,595 & 1,604 & 1,666 & 1,742 & 1,858 & 1,912 & 1,965 & 1,980 & 1,969 & 1,943 & 1,929 \\
\hline Squelette $100-200 \mu \mathrm{m}$ & 1,619 & 1,587 & 1,572 & 1,591 & 1,614 & 1,706 & 1,761 & 1,817 & 1,852 & 1,875 & 1,903 & 1,929 \\
\hline
\end{tabular}

Soient $\rho_{\mathrm{s}}^{\mathrm{a}}$ et $\rho_{\mathrm{s}}^{\mathrm{s}}$ les masses volumiques respectives de l'argile et du squelette, $A$ et $S$ les poids respectifs d'argile et de squelette dans $100 \mathrm{~g}$ de mélange, $\rho_{\mathrm{a}}$ la masse volumique de l'argile seule, on peut alors calculer par les relations suivantes :

- le volume de lacune,

$$
V_{1}=\frac{100}{\rho_{d}}-\frac{S}{\rho_{s}^{s}}-\frac{A}{\rho_{a}}
$$

- le volume de pores de l'argile,

$$
\mathrm{V}_{\mathrm{a}}=\frac{\mathrm{A}}{\rho_{\mathrm{a}}}-\frac{\mathrm{A}}{\rho_{\mathrm{s}}^{\mathrm{a}}}
$$

Les échantillons ont été soumis à une mesure de porosimétrie au mercure. Après un rappel du principe de la mesure, les problèmes particuliers rencontrés seront plus particulièrement développés.

\section{B. Porosimétrie au mercure}

\section{Principe}

Rappelons qu'elle permet d'établir une courbe cumulative des volumes de mercure entrés dans un échantillon sous l'effet de pressions croissantes.

La loi de Jurin permet de transformer ces pressions en termes de diamètre équivalent d'entrée des pores selon la relation :

$$
D=-\frac{4 \sigma \cos \theta}{P}
$$

avec $\sigma$ : tension superficielle du mercure,

$\theta$ : angle de raccordement du ménisque à la paroi,

$P$ : différence de pression de part et d'autre du ménisque,

$\mathrm{D}$ : diamètre du capillaire équivalent au pore au niveau du ménisque.

\section{Conditions de réalisation des mesures}

Un ensemble de conditions générales est rappelé par PELlERIN (1980) ; elles concernent la nature non réac- tive au mercure des échantillons, leur état de déshydratation, les conditions d'équilibre vis-à-vis de la conductivité au mercure.

Avec l'appareil utilisé ici (9300 COULTRONICS), l'échantillon est placé dans une cellule constituée d'une chambre cylindrique de $16 \mathrm{~mm}$ de diamètre et $26 \mathrm{~mm}$ de profondeur environ. Il y est soumis à un vide initial correspondant à une pression inférieure à $7.10^{-6} \mathrm{MPa}$ avant que la cellule soit remplie par le mercure. Les mesures commencent avec une pression nominale de l'ordre de $3.10^{-3} \mathrm{MPa}$ (rappelons que $1 \mathrm{MPa}=10$ bars) et la pression maximale applicable est de $206 \mathrm{MPa}$. La loi de Mariotte montre qu'à cette pression maximale, la pression de l'air piégé initialement dans l'échantillon ne dépasse pas $10^{-3} \mathrm{MPa}$. On peut donc négliger la pression de l'air piégé à toutes les étapes de mesure et ne tenir compte dans le calcul des diamètres que des pressions nominales affichées par la jauge de mesure.

Les mesures de volumes de mercure entrés dans l'échantillon s'effectuent par mesure de capacité au niveau du capillaire prolongeant la chambre. Ce capillaire est métallisé à sa périphérie et constitue donc un condensateur quand sa lumière intérieure est occupée par le mercure.

Le capillaire utilisé ici a une longueur utile de $200 \mathrm{~mm}$ et un diamètre interne de $3,2 \mathrm{~mm}$. Le volume de mercure utilisable est d'environ $1,8 \mathrm{~cm}^{3}$. La précision annoncée de la mesure de capacité étant de 1 p. 100 en pleine échelle, le volume de $1,8 \mathrm{~cm}^{3}$ est connu à mieux que $\pm 0,02 \mathrm{~cm}^{3}$.

\section{Difficultés rencontrées}

Les mesures s'effectuent d'abord dans un poste dit de «basse pression », où les pressions appliquées sont inférieures ou égales à la pression atmosphérique, puis dans un poste « haute pression » où les pressions sont transmises au mercure par l'intermédiaire d'une huile spéciale.

Dans le $1^{\text {er }}$ poste, la cellule est disposée dans le plan horizontal. Ainsi, la pression initiale subie par l'échantillon correspond, au maximum, à la hauteur 
de mercure emplissant la chambre, soit $16 \mathrm{~mm}$, ce qui correspond à un diamètre d'entrée d'environ $600 \mu \mathrm{m}$.

En haute pression, la nécessité technique d'immersion de la cellule dans l'huile impose une position verticale.

Il est alors nécessaire d'ajouter à la pression nominale la pression exercée par la colonne de mercure encore présent dans le capillaire. Cette correction est égale ou inférieure à $0,03 \mathrm{MPa}$.

Cependant, pour les échantillons présentant une entrée de mercure à des pressions inférieures à la pression atmosphérique, le fait de passer en position verticale provoque une entrée non contrôlée de mercure, les mesures de capacité en haute pression étant réalisées de manière indépendante de celles effectuées en basse pression (courbe 1, fig. 1).

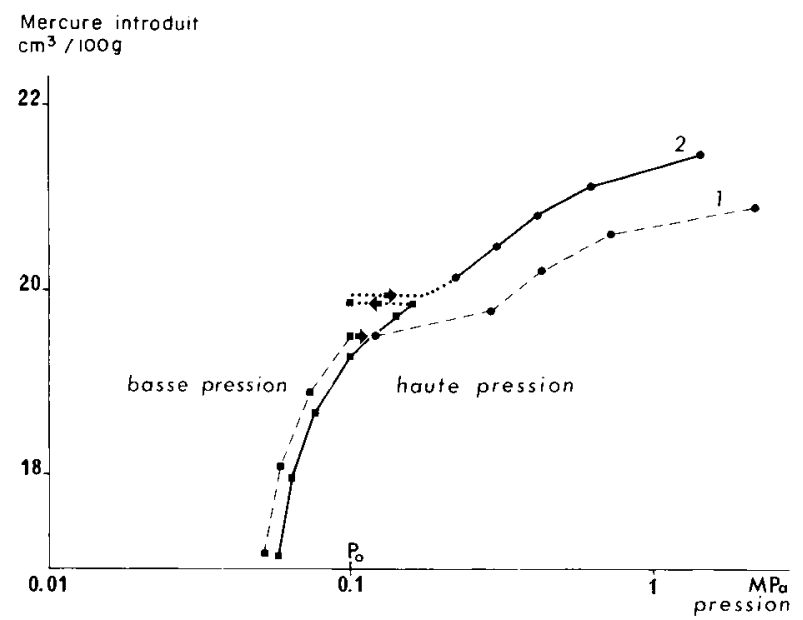

Figure 1

Raccordement des courbes d'entrée de mercure : influence du protocole suivi avant le changement de poste de mesure à $P_{0}$.

Adjustment of cumulative mercury intrusion curves : effect of the way in which pressures are applied before removing the cell from low to high pressure measurements, at $P_{0}$.

Il y a ici un problème de raccordement des 2 parties de la courbe cumulative d'entrée de mercure. Avec l'appareil utilisé, il est possible, tout en laissant la cellule dans le poste basse pression, de réaliser des pressions un peu supérieures à $0,14 \mathrm{MPa}$ en utilisant de l'azote sous pression, puis de ramener la cellule à la pression atmosphérique pour effectuer ensuite le passage en haute pression. Ceci permet de soumettre l'échantillon à une pression légèrement supérieure à celle subie lors du retournement de la cellule, tout en contrôlant les quantités de mercure ayant pénétré dans l'échantillon.

Un exemple de résultat obtenu par cette procédure est illustré à la figure 1 (courbe 2). Elle n'est satisfaisante que dans la mesure où il n'y a pas de sortie de mercure lors du retour à la pression atmosphérique, ce qui apparaît être le cas pour les mélanges de type sablo-argileux.

Un $2^{\mathrm{e}}$ problème réside dans les variations observées de capacité lors d'essais de montée en pression sur la cellule remplie de mercure (GAvigLIO, comm. pers., 1983). Ce phénomène nécessite la répétition d'essais à blanc afin de corriger les résultats bruts de mesure de capacité. Il faut noter que PELLERIN (1980) signale aussi la nécessité d'essais à blanc sur un appareil CARlo-Erba.

\section{Fiabilité des mesures en porosimétrie}

Les volumes de mercure ayant pénétré l'échantillon sont exprimés en $\mathrm{cm}^{3}$ pour $100 \mathrm{~g}$.

Les courbes cumulées de volume de mercure sont construites en fonction du diamètre d'entrée calculé selon la relation (3). Les mesures étant destinées à être comparatives et le mercure utilisé étant toujours le même (hexadistillé, Rhône-Alpes), les calculs de diamètre d'entrée sont effectués avec les constantes suivantes :

- tension superficielle du mercure : $484 \mathrm{dyne} / \mathrm{cm}$,

- angle de raccordement du ménisque : $130^{\circ}$.

PELlerin (1980) indique en effet des variations possibles de l'angle de raccordement, avec la rugosité des parois, comprises entre $130^{\circ}$ et $140^{\circ}$. Pour cette dernière valeur, il conviendrait de multiplier par 1,19 les valeurs des diamètres calculées dans cet article.

La gamme des diamètres d'entrée correspondant aux pressions appliquées s'étend de $400 \mu \mathrm{m}$ à $0,006 \mu \mathrm{m}$.

Les mesures sont réalisées à des intervalles de temps variables de manière à s'approcher d'une valeur d'entrée de mercure proche de l'équilibre pour chaque pas de pression. On a remarqué que des temps d'équilibre longs de 15 à $45 \mathrm{mn}$, sont nécessaires dans les zones de forte pénétration de mercure. Le critère retenu est d'observer une variation de capacité au plus égale à 0,01 picofarad soit $3 \cdot 10^{-4} \mathrm{~cm}^{3}$ de mercure, dans un intervalle de 1 à $2 \mathrm{mn}$. Dans la gamme des diamètres étudiée, l'obtention d'une courbe nécessite en moyenne $7 \mathrm{~h}$ pour 30 à 35 points de mesure.

La fiabilité des mesures du volume de mercure entrant dans l'échantillon sous l'effet de pressions croissantes a été testée sur 2 types d'échantillons témoins. L'un correspond à un mélange de billes de verre de $100 \mu \mathrm{m}$ environ, non poreuses, et de 20 p. 100 d'une fraction argileuse, préparé selon le protocole décrit précédemment. Le $2^{\mathrm{e}}$ est constitué par un matériau limoneux préparé industriellement (terre de faïence), homogénéisé par malaxage, découpé en cubes et porté à $900{ }^{\circ} \mathrm{C}$. Les résultats obtenus sont portés à la figure 2. La montée en pression n'étant pas automatisée, les valeurs de volume de mercure présentées ont été calculées par intrapolation linéaire entre 2 mesures successives afin d'établir une comparaison pour des valeurs identiques de diamètre d'entrée.

Les résultats obtenus sur 12 échantillons du matériau limoneux, découpés en cubes de 2,7 à $1,8 \mathrm{~g}$, montrent que l'entrée majeure de mercure, observable entre 1 et $0,5 \mu \mathrm{m}$, est marquée par une forte variabilité. A $0,5 \mu \mathrm{m}$ les valeurs extrêmes vont de 0,3 à $10,5 \mathrm{~cm}^{3} / 100 \mathrm{~g}$, la valeur moyenne se situant à $8,1 \mathrm{~cm}^{3} / 100 \mathrm{~g}$.

Cette variabilité s'atténue ensuite : à $0,4 \mu \mathrm{m}$, les valeurs extrêmes sont de 11,0 à $15,0 \mathrm{~cm}^{3} / 100 \mathrm{~g}$ et, à $0,3 \mu \mathrm{m}$, de 15,6 et $17,2 \mathrm{~cm}^{3} / 100 \mathrm{~g}$.

L'homogénéité globale des échantillons est confirmée par la convergence des valeurs observées au-delà de $0,4 \mu \mathrm{m}$ et par l'homogénéité des valeurs de masse volumique : sur 17 échantillons on trouve $\rho_{d}=1,846$ $\pm 0,002$ ce qui représente un volume de pore calculé 




Figure 2

Courbes cumulatives d'entrée de mercure.

$S=$ mélange de billes de verre $(100 \mu \mathrm{m})$ et de $20 \mathrm{p} .100$ d'argile $(0-2 \mu \mathrm{m}) ; 3$ répétitions.

$l=$ limon argileux remanié ; 12 répétitions.

Cumulative mercury intrusion curves.

$S=$ mixture of glass beads $(100 \mu \mathrm{m})$ and $20 \%$ of clay $(0-2 \mu \mathrm{m})$ 3 replica.

$l=$ remoulded silty clay loam ; 12 replica .

compris entre 18,39 et $18,50 \mathrm{~cm}^{3} / 100 \mathrm{~g}$. La variabilité observée dans la zone de forte pénétration pourrait alors dépendre du lissage de la surface des échantillons, dont le découpage a été réalisé à l'état humide.
Le matériau sablo-argileux est testé sur 3 échantillons prismatiques de 8 à $5,5 \mathrm{~g}$, découpés à l'état sec.

Dans la zone de forte pénétration de mercure (fig. 2), la variabilité observée est plus réduite que précédemment : à $15 \mu \mathrm{m}$, les volumes mesurés vont de 2,9 à $4,7 \mathrm{~cm}^{3} / 100 \mathrm{~g}$. A $100 \mu \mathrm{m}$, les volumes mesurés vont de 0,3 à $0,45 \mathrm{~cm}^{3} / 100 \mathrm{~g}$ et à $1 \mu \mathrm{m}$ de 15,4 à $15,7 \mathrm{~cm}^{3} / 100 \mathrm{~g}$.

La variabilité s'accroît à nouveau dans la partie correspondant aux diamètres de 0,1 à $0,01 \mu \mathrm{m}$, les valeurs observées variant ici de 16,3 à $17,1 \mathrm{~cm}^{3} / 100 \mathrm{~g}$.

Il ressort d'une manière générale que les volumes de mercure mesurés après le domaine de forte pénétration sont connus avec une bonne précision, l'écart relatif étant de l'ordre de 2 p. 100.

L'objet de notre étude étant principalement de comparer des bilans dans un premier temps, il n'a été réalisé qu'une seule courbe d'entrée de mercure par échantillon.

\section{RÉSULTATS ET DISCUSSION}

\section{A. Analyse des courbes d'entrée de mercure}

\section{Remarques préliminaires}

Le tableau 3 rassemble les éléments obtenus par le calcul des bilans de volume pour les 2 séries de mélanges étudiés.

Les données de porosimétrie à mercure qui vont maintenant être présentées sont discutées relativement à l'existence de 2 compartiments dans l'espace poral textural. On doit en effet schématiquement s'attendre à obtenir une $1^{\text {re }}$ entrée de mercure correspondant à l'occupation de la phase des lacunes, suivie d'une

TABLEAU 3

Volumes de pores de la phase argileuse et volumes de lacunes calculés en $\mathrm{cm}^{3}$ pour $100 \mathrm{~g}$ de mélange aux différentes teneurs en argile des échantillons.

Clay pore volume and lacunar pore volume calculated in $\mathrm{cm}^{3} / 100 \mathrm{~g}$ for clay and sand or silt mixtures, at various clay contents.

\begin{tabular}{|c|c|c|c|c|c|c|c|c|c|c|c|c|c|}
\hline Phase argileuse & $\begin{array}{l}\text { teneur pondérale } \\
\text { pores }\end{array}$ & $\begin{array}{l}10 \\
1,62\end{array}$ & $\begin{array}{l}15 \\
2,44\end{array}$ & $\begin{array}{l}20 \\
3,25\end{array}$ & $\begin{array}{l}25 \\
4,06\end{array}$ & $\begin{array}{l}30 \\
4,87\end{array}$ & $\begin{array}{l}40 \\
6,50\end{array}$ & $\begin{array}{l}50 \\
8,12\end{array}$ & $\begin{array}{l}60 \\
9,74\end{array}$ & $\begin{array}{l}70 \\
11,37\end{array}$ & $\begin{array}{l}80 \\
12,99\end{array}$ & $\begin{array}{l}90 \\
14,62\end{array}$ & $\begin{array}{l}100 \\
16,24\end{array}$ \\
\hline Phase lacune & $\begin{array}{l}\text { squelette } 2-20 \mu \mathrm{m} \\
\text { squelette } 100-200 \mu \mathrm{m}\end{array}$ & $\begin{array}{l}23,98 \\
22,68\end{array}$ & $\begin{array}{l}23,44 \\
23,22\end{array}$ & $\begin{array}{l}22,35 \\
23,11\end{array}$ & $\begin{array}{l}19,29 \\
21,64\end{array}$ & $\begin{array}{l}15,93 \\
20,04\end{array}$ & $\begin{array}{l}10,86 \\
15,28\end{array}$ & $\begin{array}{r}7,86 \\
12,03\end{array}$ & $\begin{array}{l}4,97 \\
8,87\end{array}$ & $\begin{array}{l}3,11 \\
6,41\end{array}$ & $\begin{array}{l}1,91 \\
4,33\end{array}$ & $\begin{array}{l}1,11 \\
2,13\end{array}$ & $\begin{array}{l}0 \\
0\end{array}$ \\
\hline
\end{tabular}

$2^{e}$ entrée correspondant à la pénétration du mercure dans les pores propres à la phase argileuse. L'amplitude des 2 phénomènes doit dépendre des volumes disponibles : elle doit donc diminuer quand la teneur en argile croît, pour le compartiment lacune, et croître avec la teneur en argile, pour le compartiment argile, conformément aux résultats exposés au tableau 3.

L'occupation des volumes des pores de chaque compartiment dépend des diamètres d'accès, ou seuil, de ces pores. Ainsi, les 2 phénomènes évoqués cidessus n'apparaîtront distincts que si, d'une part, l'accessibilité aux pores lacunaires ne se fait pas au travers de seuils correspondant aux seuls pores de l'argile, d'autre part, les diamètres d'entrée des pores lacunaires sont suffisamment différents de ceux de la phase argileuse.

En ce qui concerne cette dernière condition, on peut avancer, au vu des lames minces, qu'elle sera vérifiée pour les mélanges à squelette sableux 100-200 $\mu \mathrm{m}$, mais qu'une certaine confusion entre pores lacunaires et pores de l'argile peut être attendue dans le cas des mélanges à squelette de limon 2-20 $\mu \mathrm{m}$.

\section{Les courbes d'entrée de mercure des mélanges à squelette sableux}

Considérons tout d'abord de manière globale la forme des courbes obtenues sur les mélanges à squelette sableux, présentées à la figure 3 . 




Figure 3

Courbes cumulatives d'entrée de mercure dans les mélanges sables (100-200 $\mu \mathrm{m})$-argile.

En paramètre : teneur pondérale en argile.

Cumulative mercury intrusion curves for sand $(100-200 \mu \mathrm{m})$ and clay mixtures.

Parameter : clay content.

Pour la majorité des échantillons, correspondant aux teneurs en argile de 10 à 80 p. 100, les courbes présentent une $1^{\text {re }}$ phase d'entrée de mercure que l'on peut situer entre 100 et $1 \mu \mathrm{m}$ selon les mélanges.

L'accroissement de la teneur en argile ne semble être relié à un abaissement des valeurs des diamètres auxquelles se produit cette $1^{\text {re }}$ phase d'entrée qu'avec les mélanges contenant $40 \mathrm{p}$. 100 et plus d'argile. $\mathrm{Au}$ contraire, les courbes d'entrée de mercure sont presque confondues pour les échantillons contenant de 10 à 30 p. 100 d'argile.

Cette $1^{\text {re }}$ phase est suivie d'un palier, situé entre 1 et $0,1 \mu \mathrm{m}$ environ, auquel succède une $2^{\mathrm{e}}$ phase d'entrée de mercure qui se situe cette fois-ci à des diamètres très voisins pour tous les mélanges.

Le mélange à 90 p. 100 d'argile ne se différencie pas nettement de l'échantillon d'argile pure; la phase d'entrée majeure de mercure se situe pour ce mélange et pour l'échantillon d'argile à des diamètres inférieurs à $0,1 \mu \mathrm{m}$.

Du point de vue qualitatif, les courbes obtenues sur ces mélanges paraissent rendre compte de l'existence de lacunes, marquée par la $1^{\text {re }}$ phase d'entrée de mercure. La fin de leur remplissage par le mercure correspondrait à la présence du palier. Le mercure ne pénétrerait dans le compartiment poreux argileux, bien distinct du précédent, qu'à travers des pores de diamètre inférieur à $0,1 \mu \mathrm{m}$.

On peut remarquer, en effet, que les quantités de mercure entrées à partir d'un diamètre de $0,1 \mu \mathrm{m}$ croissent avec la teneur en argile des mélanges et qu'en pratique il ne rentre de mercure dans l'échantillon d'argile qu'à partir de cette même valeur de diamètre.

Par ailleurs, les quantités de mercure entrées, correspondant aux paliers, décroissent fortement en règle générale lorsqu'augmente la teneur en argile. Toutefois, cette tendance n'est pas vérifiée pour le mélange à 10 p. 100 d'argile. L'ordre de classement des paliers des courbes est cependant bien le même que celui des valeurs calculées des pores lacunaires (tabl. 3).

\section{Les courbes d'entrée de mercure des mélanges à squelette limoneux}

Les courbes relatives aux mélanges à squelette limoneux (fig. 4) montrent une $1^{\text {re }}$ phase d'entrée de mercure à des valeurs de diamètre bien inférieures à celles observées précédemment, puisqu'on peut les situer entre 5 et $0,1 \mu \mathrm{m}$ pour les mélanges où l'argile varie de 10 à 60 p. 100 .

L'accroissement de la teneur en argile s'accompagne encore d'un déplacement de la $1^{\text {re }}$ phase d'entrée de mercure vers des valeurs de plus en plus petites de diamètre pour les mélanges contenant plus de $25 \mathrm{p} .100$ d'argile, les courbes restant très proches entre elles pour les mélanges contenant moins de 25 p. 100 d'argile. Cette $1^{\text {re }}$ phase n'est pas suivie d'un palier, mais on peut constater, pour les mélanges contenant 25 p. 100 et plus d'argile, une $2^{e}$ phase d'entrée qui se manifeste ici aussi pour des diamètres très voisins et inférieurs à $0,1 \mu \mathrm{m}$. Enfin, on peut noter la grande similitude entre la courbe à 90 p. 100 d'argile et celle obtenue sur l'argile seule.



Figure 4

Courbes cumulatives d'entrée de mercure dans les mélanges limon (2-20 $\mu \mathrm{m})$-argile.

En paramètre : teneur pondérale en argile.

Cumulative mercury intrusion curves for silt $(2-20 \mu \mathrm{m})$ and clay mixtures.

Parameter : clay content 
D'une manière générale, on peut observer ici que les courbes se classent en fonction de la teneur en argile, les volumes de mercure introduits diminuant quand la teneur en argile croît.

Par rapport aux précédentes, ces courbes posent le problème de la différenciation entre compartiment poreux lacunaire et compartiment poreux argileux. Nous nous servirons des résultats obtenus sur les mélanges à squelette sableux pour définir une valeur de diamètre d'entrée de mercure dans la phase argileuse, dont nous discuterons si elle peut être appliquée aux mélanges à squelette limoneux en confrontant les données obtenues par bilan volumique (tabl. 3) aux mesures de porosimétrie.

\section{B. Confrontation des données porosimétriques aux données calculées}

Pour établir la limite entre compartiments poreux lacunaires et argileux, il est nécessaire de définir le diamètre des pores marquant le début de la pénétration du mercure dans la seule phase argileuse.

Ceci revient à transposer l'hypothèse de la constante de la masse volumique de l'argile à celle de la constance du spectre de distribution des pores de l'argile dans tous les échantillons.

Il est toujours difficile de définir un point singulier sur une courbe.

La démarche alors suivie est de rechercher, sur les courbes bien différenciées des mélanges à squelette sableux, avec quelle même valeur d'un diamètre $d_{a}$ de début d'entrée du mercure dans la phase argile on obtient le meilleur ajustement entre le volume de mercure mesuré jusqu'à $\mathrm{d}_{\mathrm{a}}$ et le volume calculé de lacune. On discutera ensuite de la validité du choix de cette valeur de $\mathrm{d}_{\mathrm{a}}$ pour les mélanges à squelette limoneux.

\section{Echantillons à squelette sableux}

Le tableau 4 rassemble les résultats du test de corrélation linéaire entre volumes calculés et mesurés, obtenus avec 11 couples de valeurs, $d_{a}$ étant compris entre 1 et $0,01 \mu \mathrm{m}$.

\section{TABLEAU 4}

Résultats du test de corrélation linéaire entre volume de lacune mesuré et calculé, pour les mélanges à squelette sableux $\left(\mathrm{cm}^{3}\right.$ pour $\left.100 \mathrm{~g}\right)$.

Linear correlation between measured and calculated lacunar pore volume of the sandy skeleton samples.

$y$ : volume de mercure entré dans l'échantillon jusqu'au diamètre limite choisi.

Mercury volume intruded to boundary diameter value.

$x$ : volume de lacune calculé.

Calculated lacunar volume.

$d_{a}$ : diamètre limite en $\mu \mathrm{m}$.

Boundary diameter value, in $\mu m$.

\begin{tabular}{lccc}
\hline \hline \multicolumn{1}{c}{} & Equation de la droite & $\mathrm{r}^{2}$ & $\begin{array}{c}\text { Volume de mercure } \\
\text { entré dans l'échantillon } \\
\text { d'argile pure jusqu’à } \mathrm{d}_{\mathrm{a}}\end{array}$ \\
\hline $\mathrm{l}$ & $\mathrm{y}=1,080 \mathrm{x}-1,65$ & 0,990 & 0 \\
0,1 & $\mathrm{y}=1,067 \mathrm{x}-1,11$ & 0,989 & 0,2 \\
0,05 & $\mathrm{y}=1,039 \mathrm{x}-0,46$ & 0,988 & 0,3 \\
0,01 & $\mathrm{y}=0,964 \mathrm{x}+2,22$ & 0,992 & 3,3 \\
\hline \hline
\end{tabular}

Compte tenu du fait que la part expliquée de la variance du volume mesuré au mercure reste pour chaque valeur de $d_{a}$ toujours de l'ordre de 99 p. 100, les critères discriminant du choix de $d_{a}$ restent les valeurs de la pente et de l'ordonnée à l'origine qui doivent respectivement être proches de 1 et de 0 , ainsi que le volume de mercure entré dans l'échantillon d'argile pure, qui doit rester proche de zéro pour le diamètre $d_{a}$ choisi.

C'est en définitive la relation obtenue avec un diamètre de $0,05 \mu \mathrm{m}$ qui vérifie le mieux ces critères. La figure 5 illustre la distribution des points alors obtenue.

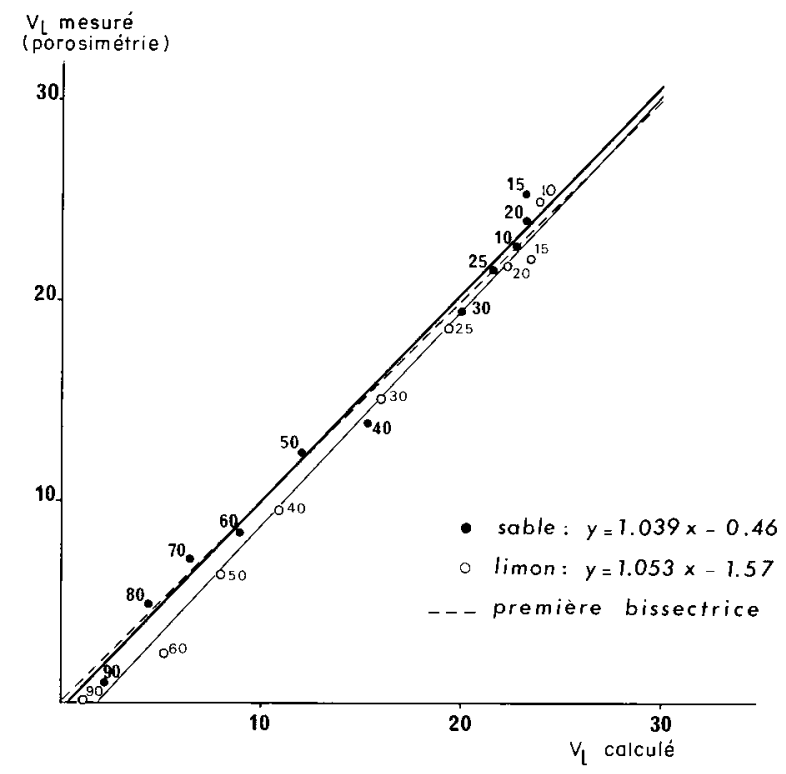

Figure 5

Comparaison des volumes de lacune mesurés au porosimètre à mercure et calculés.

En paramètre: teneur en argile.

Comparison between lacunar pore volume values measured by mercury porosimetry and calculated.

Parameter : clay content.

En particulier, la quantité de mercure entrée dans l'échantillon d'argile à cette valeur de $d_{a}$ représente seulement 3,3 p. 100 de la quantité totale ayant pénétré jusqu'au diamètre de $0,006 \mu \mathrm{m}$. A la valeur de $d_{a}$ de $0,01 \mu \mathrm{m}$, la proportion de mercure introduite dans cet échantillon est déjà de 36,3 p. 100.

On peut donc admettre pour ces échantillons qu'à la valeur de $0,05 \mu \mathrm{m}$, la phase de lacune est occupée à 100 p. 100 par le mercure.

En ce qui concerne les pores de la phase argileuse, il apparaît qu'ils ne sont jamais saturés quand le diamètre de $0,006 \mu \mathrm{m}$ est atteint.

Appelons $\mathrm{T}$ le taux de remplissage, c'est-à-dire le rapport du volume de mercure ayant pénétré entre $d_{a}$ et $0,006 \mu \mathrm{m}$ au volume total de pore calculé dans $100 \mathrm{~g}$ de mélange (tabl. 3 ).

Pour la valeur de $d_{a}$ de $0,05 \mu \mathrm{m}$, la valeur de $T$ est en moyenne de $51 \mathrm{p}$. 100 . Les valeurs extrêmes sont de 44 et 66 p. 100 pour les échantillons contenant respectivement 50 et 70 p. 100 d'argile, la valeur trouvée pour l'argile pure est de $56 \mathrm{p} .100$. Compte tenu de la présence d'un palier sur les courbes d'entrée de mercure, la valeur de $T$ n'est pas sensiblement modifiée 
par le choix d'une valeur plus forte pour $d_{a}$. Ainsi, à $\mathrm{d}_{\mathrm{a}}$ choisi à $0,1 \mu \mathrm{m}$, la valeur de $\mathrm{T}$ est encore en moyenne de $53 \mathrm{p} .100$.

Cette relative constance du taux de remplissage des pores de la phase argileuse par le mercure dans les différents mélanges est aussi en accord avec l'hypothèse d'une distribution de la taille des pores relativement homogène dans les échantillons et indépendante de la quantité de squelette sableux associée.

\section{Echantillons à squelette limoneux}

Appliqué maintenant aux valeurs obtenues sur échantillons à squelette limoneux, le test de corrélation précédent (voir aussi tabl. 4) conduit au résultat suivant, pour $d_{a}=0,05 \mu \mathrm{m}$ :

$$
\mathrm{y}=1,076 \mathrm{x}-2,05 \quad \mathrm{r}^{2}=0,993 ; 9 \text { couples de valeurs. }
$$

Par rapport à la relation précédente, la droite obtenue s'écarte davantage de la $1^{\text {re }}$ bissectrice. Les valeurs de la pente et de l'ordonnée à l'origine ne sont pas améliorées quand on passe à une valeur de $0,1 \mu \mathrm{m}$ pour $\mathrm{d}_{\mathrm{a}}$.

En ce qui concerne la phase argileuse, le calcul des taux de remplissage $T$, pour $d_{a}=0,05 \mu \mathrm{m}$, conduit à une valeur moyenne plus élevée que précédemment, 65 p. 100 , les valeurs les plus fortes étant obtenues sur les mélanges les moins riches en argile : 86 et 71 p. 100 avec les échantillons contenant respectivement 10 et 15 p. 100 d'argile. Cette tendance pourrait résulter soit d'une modification de la distribution des pores de la phase argileuse vers des valeurs plus élevées de diamètre, soit d'une confusion entre les pores lacunaires et les pores de l'argile.

Pour tenter d'amorcer la discussion des conséquences de ces hypothèses de travail, il est admis ici qu'une variation de distribution des pores de l'argile ne peut être disjointe d'une variation corollaire au niveau de la masse volumique de l'argile.

Dans l'hypothèse d'une modification de la distribution des pores de l'argile, on peut, en $1^{\text {re }}$ approximation, admettre qu'un enrichissement en pores plus grossiers ait pour conséquence une diminution de la valeur de la masse volumique $\rho_{\mathrm{a}}$ de la phase argileuse. Cela permettrait de réduire, d'après la relation (1), la valeur du volume $V_{1}$ attaché aux pores lacunaires. Cependant, cette variation de $\rho_{\mathrm{a}}$ dépend vraisemblablement d'une interaction argile-squelette et doit être d'autant moins importante que la teneur en argile croît. Dans ces termes, une diminution de $\rho_{a}$ ne peut rendre compte alors du fait que les valeurs calculées de volume de lacune (fig. 5) sont, pour les teneurs en argile moyennes et fortes, systématiquement supérieures aux volumes mesurés par porosimétrie.

$\mathrm{Au}$ niveau de la porosimétrie, la même hypothèse implique une entrée de mercure dans la phase argileuse avant le seuil de $0,05 \mu \mathrm{m}$. Les valeurs de $V_{1}$ mesurées au mercure seraient donc déjà surévaluées, ce qui ne va pas non plus dans le sens souhaité pour rapprocher les valeurs mesurées et calculées de $V_{1}$.

La $2^{\mathrm{e}}$ hypothèse présente 2 aspects. Le $1^{\mathrm{er}}$ revient à admettre que la confusion porte au niveau des seuils d'entrée les plus petits des pores lacunaires et les plus grossiers des pores de l'argile. Mais ce phénomène de confusion doit tendre à diminuer quand augmente la teneur en argile et que le nombre de pores lacunaires que l'on peut admettre proportionnel au nombre de grains de limon se réduit. Le $2^{\mathrm{e}}$ aspect est celui de l'accessibilité des lacunes. On peut en effet avancer que, du fait de la petite taille du squelette, la probabilité d'isolement des lacunes dans la phase argileuse doit d'abord croître avec la teneur en argile, puis décroître aux très fortes teneurs par disparition des lacunes. Cela peut expliquer alors l'écart observé èntre volume de lacune calculé et mesuré, sur les mélanges contenant de 25 à 60 p. 100 d'argile.

Par exemple, pour le mélange à 50 p. 100 d'argile, cet écart représente $2,3 \mathrm{~cm}^{3}$ par $100 \mathrm{~g}$, soit presque le tiers du volume calculé de lacune.

Cependant, l'ensemble de cette $2^{\mathrm{e}}$ hypothèse implique que, tout comme pour la mesure du volume des pores de l'argile, le volume de lacune confondu avec celui des pores de l'argile, n'est occupé que partiellement par le mercure au cours de la mesure de porosimétrie. La correction du volume de lacune à partir des données expérimentales risque donc de sous-évaluer encore le volume de lacune.

Ainsi, si $\mathrm{V}_{\mathrm{a}}$ représente le volume de mercure entré dans l'échantillon d'argile entre 0,05 et $0,006 \mu \mathrm{m}$, le produit $\mathrm{V}_{\mathrm{a}}$. A est une estimation du volume de mercure entré dans le même intervalle dans un mélange, en l'absence de lacunes isolées. Soit $\mathrm{V}$ le volume de mercure mesuré dans le même intervalle sur le mélange considéré, la différence $\mathrm{V}-\mathrm{V}_{\mathrm{a}}$. A représente une estimation du volume de lacunes isolées.

Après cette correction, la corrélation entre volume de lacune calculé et volume mesuré devient, avec $\mathrm{d}_{\mathrm{a}}=0,05 \mu \mathrm{m}$ :

$$
\mathrm{y}=1,053 \mathrm{x}-1,57 \quad \mathrm{r}^{2}=0,995 ; 9 \text { couples de valeurs. }
$$

Cette relation est améliorée par rapport à la précédente essentiellement au niveau de l'ordonnée à l'origine mais on peut penser que l'écart qui subsiste avec la $1^{\text {re }}$ bissectrice est le fait de la sous-évaluation invoquée plus haut.

\section{CONCLUSION}

Cet ensemble de résultats montre que les données de la porosimétrie au mercure et, en particulier, la forme générale des courbes d'entrée de mercure sont en accord avec le modèle du double compartiment de porosité développé à la suite des calculs de bilan de volume.

Ce modèle se situe à l'échelle de l'assemblage des constituants squelette-argile étudié sur des mélanges après séchage. Il apparaît validé, d'une part, pour une très large gamme de teneur en argile, d'autre part, pour des squelettes sableux ou limoneux.

Ces résultats étaient considérés comme acquis, au moins qualitativement, sur les mélanges à squelette sableux. L'usage de la porosimétrie au mercure a permis de les étendre aux mélanges à squelette limoneux.

D'une manière générale, il est apparu possible d'établir une relation entre une approche globale de la porosité texturale par des mesures de masse volumique et une approche quantitative par porosimétrie.

Cette confrontation nous a, en particulier, appris que le $1^{\text {er }}$ compartiment poreux, correspondant aux lacunes, est constitué de lacunes qui sont directement 
accessibles au mercure par des pores de taille supérieure à celle résultant de l'assemblage des phyllites de la phase argileuse. Deux cas semblent devoir être distingués : celui des mélanges à teneur en argile faible à moyenne où la phase d'entrée de mercure semble dépendre davantage de la taille du squelette que de la teneur en argile et celui des mélanges à teneur en argile moyenne à forte où elle se produit pour des diamètres décroissants quand augmente la teneur en argile.

Cette distinction rejoint celle établie par CHRETIEN (1979), quant à la variation de la morphologie des lacunes observée sur des mélanges sable-argile, entre mélanges riches en squelette et riches en argile.

Un certain nombre d'éléments quantitatifs reste encore à acquérir pour l'établissement d'un modèle d'espace poral de ces mélanges.

Ce sont, par exemple, des données sur la forme des lacunes. Celles obtenues par CHRETIEN sur les seuls mélanges à 20 p. 100 d'argile montrent que leur diamètre estimé sur lame mince est voisin de celui du squelette utilisé. On peut donc penser, compte tenu de nos résultats, que les pores d'accès aux lacunes sont petits par rapport aux diamètres des lacunes. A cet égard, une étude particulière d'un mélange de sable grossier et de 15 p. 100 d'argile (FIES \& ZIMMER, 1982) devait montrer que le rapport entre le diamètre de la lacune et son accès était de l'ordre de 10.

A ces teneurs en argile et à cet état de dessiccation, les lacunes constitueraient donc une succession de ventres et de nœuds, selon un modèle semblable à celui élaboré par CHATZIS \& DULLIEN (1982) pour des grès.

Par ailleurs, il reste encore difficile d'accéder directement, à partir des courbes d'entrée de mercure, à la connaissance des distributions des pores lacunaires et de leurs seuils d'accès.

En effet, d'après la théorie de la percolation, à un diamètre donné dans la phase d'entrée de mercure, les lacunes présentant un seuil d'accès de ce diamètre ne sont pas toutes accessibles directement. Le volume de mercure introduit ne correspond alors qu'à une occupation partielle des lacunes occupables. Les travaux de GANOULIS (1974), LENORMAND (1981) font clairement apparaître qu'il s'agit d'un cas général qu'il faut aborder à travers des hypothèses portant sur la forme des pores et en particulier leur mode d'anastomose.

Cette modélisation du système poreux à l'état sec peut servir de base au passage à des systèmes étudiés à des états hydriques variés. Il est probable que le gonflement de la phase argileuse peut modifier la géométrie et la distribution des seuils d'accès aux lacunes.

Cependant, le résultat des calculs de bilan de volume réalisés sur échantillons réhumectés (FIES, 1971) montre qu'il y a conservation du double compartiment de porosité jusqu'à des teneurs élevées en argile.

Vis-à-vis des phénomènes de transferts hydriques, il est peut être utile de pouvoir distinguer, dans la teneur en eau totale, le volume concerné par les pores lacunaires de celui occupant les pores de l'argile, surtout si les seuils d'accès à ces domaines conservent un caractère bimodal suffisamment marqué. Quelques essais réalisés sur des mélanges argile-limon montrent que cela est probable (FIES, 1983).

Par ailleurs, vis-à-vis des phénomènes de transfert dans la phase argileuse, il apparaît nécessaire de tenir compte, dans le cas de squelette fin associé, d'une accessibilité et d'une surface d'échange beaucoup plus grandes de la phase argile aux faibles et moyennes teneurs en argile.

Enfin, les correspondances observées entre mélanges binaires et matériaux naturels (FIES \& STENGEL, $1981 b$ ) permettent de bien augurer de l'application des données obtenues sur systèmes poreux simplifiés aux couches de sol remaniées de la surface.

Reçu le 7 novembre 1983 Accepté le 24 mai 1984.

\section{RÉFÉRENCES BIBLIOGRAPHIQUES}

Chatzis I., Dullien F. A. L., 1982. Mise en œuvre de la théorie de la percolation pour modéliser le drainage des milieux poreux et la perméabilité relative au milieu non mouillant injecté. Rev. Inst. fr. Pétrole, 36 (3), 183-205.

Chretien J., 1979. Etude expérimentale de la porosité de mélanges sables-argile. Aspects micromorphologiques. Sci. Sol, 4, 333-353.

Fies J. C., 1971. Recherche d'une interprétation texturale de la porosité des sols. Ann. agron., 22 (6), 621-653.

Fies J. C., 1978. Porosité du sol : étude de son origine texturale. Thèse d'Etat, Univ. Strasbourg 1, $150 \mathrm{p}$.

Fies J. C., 1983. Répartition du volume des pores dans des assemblages argile-squelette à différents états hydriques : étude expérimentale et modélisation. Colloque Polono-Français, Lubin (à paraître).

Fies J. C., Stengel P., 1981a. Densité texturale de sols naturels. I. Méthode de mesure. Agronomie, 1 (8), 651-658.

Fies J. C., Stengel P., 1981b. Densité texturale de sols naturels. II. Eléments d'interprétation. Agronomie, 1 (8), 659-666.

Fies J. C., Zimmer D., 1982. Etude expérimentale de modifications de l'assemblage textural d'un matériau sablo-argileux sous l'effet de pressions. Bull. groupe fr. Humidité neutronique, 12, 39-55.
Ganoulis J., 1974. Remplacement d'un fluide par un autre dans des domaines de géométrie aléatoire ou non cylindrique. Première partie : 1-120 in Thèse d'Etat, Univ. Toulouse 3, $400 \mathrm{p}$.

Lenormand R., 1981. Déplacements polyphasiques en milieux poreux sous l'influence des forces capillaires. Etude expérimentale et modélisation de type percolation. Thèse d'Etat, Institut nat. polytechnique de Toulouse, $330 \mathrm{p}$.

Monnier G., Stengel P., Fies J. C., 1973. Une méthode de mesure de la densité apparente de petits agglomérats terreux. Application à l'analyse des systèmes de porosité du sol. Ann. agron., 24 (5), 533-545.

Pellerin F. M., 1980. La porosimétrie au mercure appliquée à l'étude géotechnique des sols et des roches. Bull. Liais. Lab. des Ponts et Chaussées, 106, 105-116.

Stengel P., 1981. Relations entre le retrait et le potentiel de l'eau dans des mélanges smectite-limon. C. R. Acad. Sci., Paris, 293, 465-468.

Tessier D., 1975. Recherches expérimentales sur l'organisation des particules dans les argiles. Thèse C.N.A.M., Paris, 231 p. 